\title{
Surgical Management of an Inflammatory External Root Resorptive Defect Using Mineral Trioxide Aggregate - A Case Report
}

\author{
Dr. Geeta I B ${ }^{1}$, Dr. Lynn Lilly Varghese ${ }^{2}$, Dr. P Sindhu Padmanabha ${ }^{3}$ \\ ${ }^{1}$ (Professor \& HOD) Department of Conservative Dentistry and Endodontics, Rajarajeswari Dental College \& Hospital, No.12Ramohalli \\ cross, Mysore Road, P.O - Kumbalagodu.Pin 560074. Bangalore, Karnataka, India \\ ${ }^{2}$ Postgraduate student, Department of Conservative Dentistry and Endodontics, Rajarajeswari Dental College \&Hospital, No.12 Ramohalli \\ cross, Mysore Road, P.O - Kumbalagodu.Pin 560074. Bangalore, Karnataka, India \\ ${ }^{3}$ Postgraduate student, Department of Conservative Dentistry and Endodontics, Rajarajeswari Dental College \& Hospital, No.12 Ramohalli \\ cross, Mysore Road, P.O - Kumbalagodu.Pin 560074. Bangalore, Karnataka, India
}

\begin{abstract}
Root resorption is a pathological process which tends to occur following a wide range of mechanical or chemical stimuli such as infection, pressure, trauma or orthodontic tooth movement. Although it is predominantly detected by radiography, in some cases root resorption may be identified by clinical symptoms i.e. pain, swelling and mobility of the tooth. Treatment alternatives are case dependent and aim at the removal of the cause and the regeneration of the resorptive lesion. This case report describes the diagnosis and treatment of an inflammatory external root resorptive (IERR) defect in relation to 21 of a 52 year old male patient. Following endodontic retreatment, the resorption was subsequently sealed surgically with mineral trioxide aggregate (MTA). The six month follow-up demonstrated no pathologic changes on clinical and radiographic examination.
\end{abstract}

Keywords: Inflammatory external root resorption, mineral trioxide aggregate, root perforation

\section{Introduction}

Resorption of the root of a permanent tooth is a pathological process that can occur inside the tooth (internal resorption), or on the outer surface of the tooth (external root resorption) and can ultimately lead to loosening of the tooth and its early loss. External root resorption (ERR) occurs when the cementoblastic layer or tooth tissue on the root surface are either damaged or removed [1]. Resorption has been linked with multinucleated giant cells contiguous to granulation tissue of pulpal origin [2].External root resorption tends to occur more frequently in patients aged between 21 and 30 years $(28.40 \%)$ and is more common in females $(59.04 \%)$ than males [3].Trauma, previous periodontal surgery, pressure from adjacent unerupted teeth and pathological conditions such as tumors as well as tooth re-implantation have all been implicated as etiological factors [3], [4]. These factors cause damage to the root, resulting in denuded areas on the root surface which are chemotactic to phagocytes. This inflammatory response exacerbates in the presence of bacteria and their byproducts inside the root canal system and dentinal tubules after pulp necrosis along with the absence of any protection by the cementum barrier. The resorptive process is maintained by bacterial products from the infected root canal which provides the necessary continuous stimulation for the resorbing cells, resulting in formation of larger tooth surface defects [5], [6].

Diagnosis should be based on a combination of radiographic and clinical examination. Early diagnosis is a critical factor in the management of IERR since the sooner treatment is initiated; the less severe will be the long-term consequences of resorption [7].However, IERR is an asymptomatic lesion that is difficult to diagnose and treat. Clinically the affected teeth are typically asymptomatic in the early period of the disease process. However, as IERR progresses, the teeth may become symptomatic, and periradicular abscesses may develop with increasing tooth mobility.

Intraoral radiographs of such lesions usually reveal radiolucencies near the external root surfaces adjacent to the bone with uneven root surface outlines. Radiographs obtained at different angles may be useful in determining the affected areas on the root [8], [9].However, apical shortening, lateral or cervical root gaps, enlargement of root canal and external root radiolucencies are not detectable on radiographs at their early stages due to the limitations of this 2-dimensional method. Hence, the introduction of high resolution 3- dimensional imaging into dental diagnoses through the usage of cone beam computed tomography (CBCT) has made the accurate detection of root surface lesions possible and easy. Various studies in literature report the high accuracy levels of CBCT in the detection of IERR lesions when compared with conventional radiographic techniques. Thus CBCT was used in this case in order to determine the exact extension of the resorptive lesion [10].

Mineral trioxide aggregate (MTA) has been proposed as a favorable perforation repair material with its superior sealing ability, biocompatibility, fibroblastic stimulation and antimicrobial activity [11]. In conjunction with being sterile, radiopaque, and dimensionally stable the material is not sensitive to moisture and blood contamination. Perforated roots treated with MTA showed a noninflammatory tissue layer and less leakage when compared with perforations repaired with amalgam, intermediate restorative material, zinc oxide-eugenol, and SuperEBA by using both dye and bacteria leakage methods. These favorable properties render MTA a suitable material for the management of tissue damage caused by IERR [7]. 


\section{International Journal of Science and Research (IJSR) \\ ISSN (Online): 2319-7064}

Index Copernicus Value (2013): 6.14 | Impact Factor (2014): 5.611

The present case report describes the diagnosis and retreatment followed by surgical management of an external inflammatory resorptive defect present on a left central incisor using MTA as the sealing material.

\section{Case Report}

A 52 old male patient reported to the Department of Conservative Dentistry and Endodontics, Rajarajeswari Dental College and Hospital, Bengaluru with the chief complaint of pain on chewing and recurrent swelling in relation to the labio-gingival aspect of the upper front tooth region since 6 months. Patient gave a history of trauma to the anterior tooth region 19 years back after which he had sought treatment. Root canal treatment was done in relation to 11 and 21 following which the tooth remained asymptomatic for a period of 10 years. Patient was a diabetic and was under medication for the same. On clinical examination 11 and 21 were discoloured and were sensitive to percussion. 21 showed mild mobility with normal probing depth. Periapical radiographs confirmed previous root canal treatment in relation to the central incisors in which both the root canal spaces were inadequately obturated .A diffuse periapical radiolucency was present in relation to 11 . Furthermore, extensive lateral (distal) resorption was noticed in relation to the cervical and middle third of the root of 21 along with mild bone loss adjacent to the resorptive lesion. Patient was also referred for CBCT scanning to confirm the extent of the resorptive defect and to check for any root surface perforations. CBCT images revealed that the size and extent of the root defect on 21 was underestimated and were actually larger than what was visible on the radiograph. The extent of the lesion was clearly visible in the axial and transverse slices in the cervical to mid-root region.

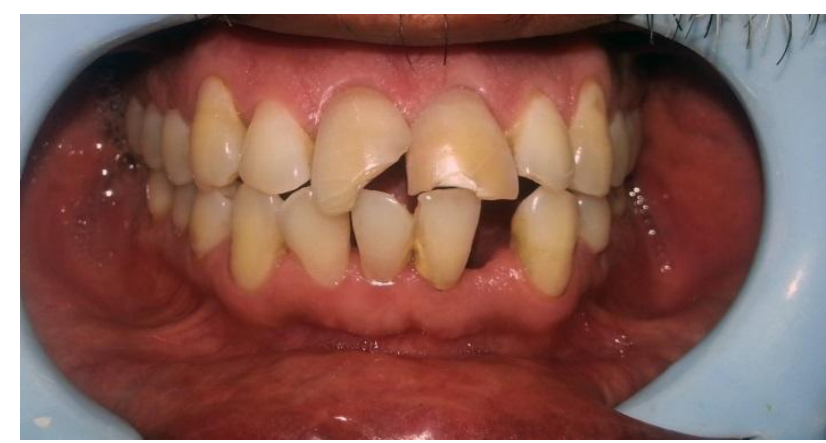

Figure 1: Preoperative labial surface view of maxillary left central incisor

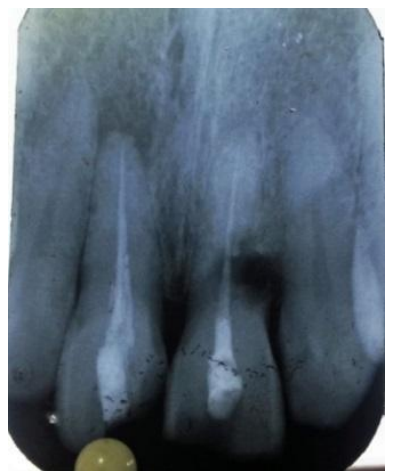

Figure 2: Preoperative IOPA radiograph

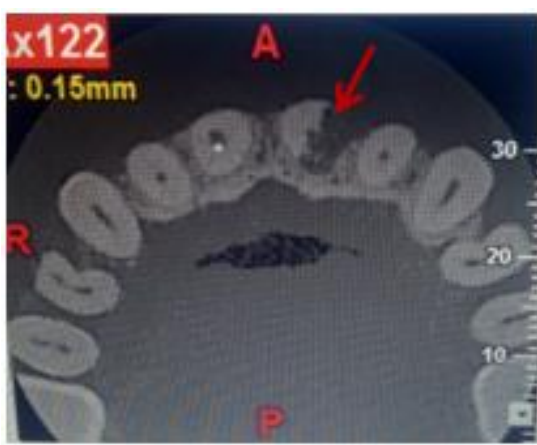

Figure 3: Axial section of CBCT of 21 depicting radiolucency on the cervical root level

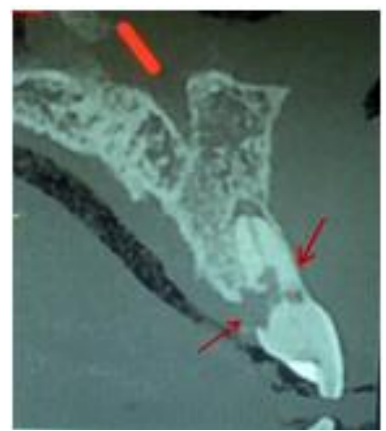

Figure 4: Transverse slice CBCT image of 21 showing the labio-palatal extent of resorptive lesion and loss of labial cortical bone

Considering the amount and severity of the resorption, endodontic retreatment in conjunction with surgical debridement and restoration of the resorption lacuna with MTA was the treatment planned in order to arrest the resorptive process occurring along the length of the root.Consent was obtained from the patient. Endodontic access was established by removal of the previous temporary access filling. Previous guttapercha filling was removed using Hedstrom files with Endosolv-E (Septodont, Inc., New castle, Delaware, USA).During the retreatment procedure, severe bleeding on root canal instrumentation was noted in relation to 21 , which was due to the presence of a perforation defect along with granulation tissue. A radiograph was taken to ensure complete removal of gutta-percha which showed uneven radiolucent regions along the external borders from the cervical to mid third level of the root, and the outline of original root canal was completely visible. This was highly suggestive of external root resorption. Working length was determined radiographically and canal preparation was completed in 11 and 21 to establish an apical stop of a 60 size K-file (Mani Inc, Japan). The root canal was irrigated between each file with $2 \%$ chlorhexidine and sterile saline by using a long needle alternatively. The canal was dried with absorbent paper points and filled with Calcium hydroxide intra canal medicament (Ultracal, South Jordan). The access cavity was sealed with a temporary restorative material (Cavit-G, 3M ESPE, St. Paul, MN, USA). After 2 weeks, the intracanal medicament was flushed out with distilled water and $1 \%$ sodium hypochlorite (Novo Dental Products Pvt. Ltd. Mumbai, India)and copious saline irrigation. 2\% chlorhexidine was used as the final irrigant and flushed with saline. The canal was dried with absorbent paper points. 11 was obturated

\section{Volume 5 Issue 1, January 2016}




\section{International Journal of Science and Research (IJSR) \\ ISSN (Online): 2319-7064}

Index Copernicus Value (2013): 6.14 | Impact Factor (2014): 5.611

using guttapercha and Endoflas sealer by lateral condensation technique. Since 21 continued to exhibit significant bleeding from within the canal, it was decided to obturate the root canal in conjunction with the surgical repair of root defect.

During the surgical procedure, local anesthesia was administered following which an intra-sulcular incision was made from the distal surface of maxillary right lateral to the distal surface of maxillary left lateral. Two vertical releasing incisions were given on both ends and a labial full-thickness mucoperiosteal flap was elevated. A circular resorptive area was clearly visible on the distal aspect of the root in relation to 21 extending labio-palatally along the proximal surface of the root. The defect housed granulation tissue which was curetted and irrigated liberally with sterile saline. After the surgical field was cleaned, the flap was placed back. The root canal of 21 was irrigated copiously with $2 \%$ chlorhexidine and sterile saline. A size 60 master cone radiograph was taken, and the root canal was obturated with gutta-percha and Endoflasby using lateral condensation technique at the same appointment. Following this, the flap was rereflected, and the defect area was rinsed with sterile saline solution. The resorption site was subsequently sealed with MTA (Angelus, Londrina, Brazil) .The flap was repositioned without tension and sutured interproximally with non-absorbable sutures (Silk; Dogsan, Turkey). The access cavities were restored permanently with glass ionomer cement (Ketac Molar, Easymix 3M ESPE).After the surgery, the patient was prescribed analgesics, amoxicillin $500 \mathrm{mg}$ thrice a day for 1 week and $0.2 \%$ chlorhexidine mouthwash $15 \mathrm{~mL}$ twice a day for 2 weeks. The patient was symptom free 1 week after the surgery, and the sutures were removed.

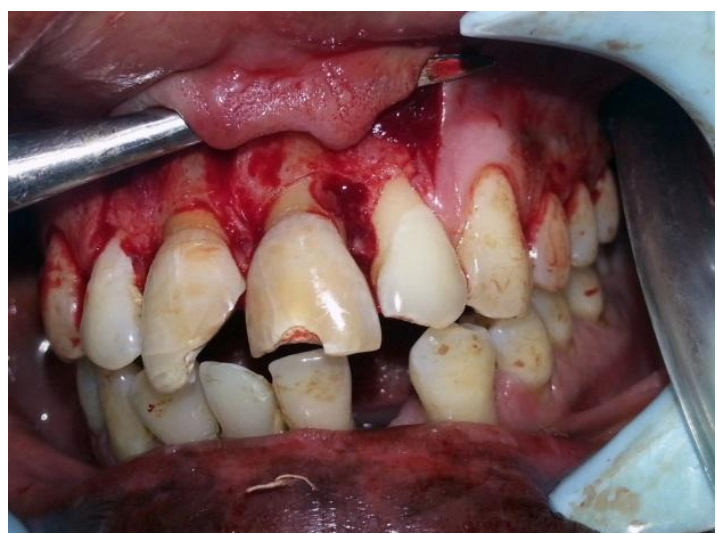

Figure 5: Full thickness mucoperiosteal flap raised

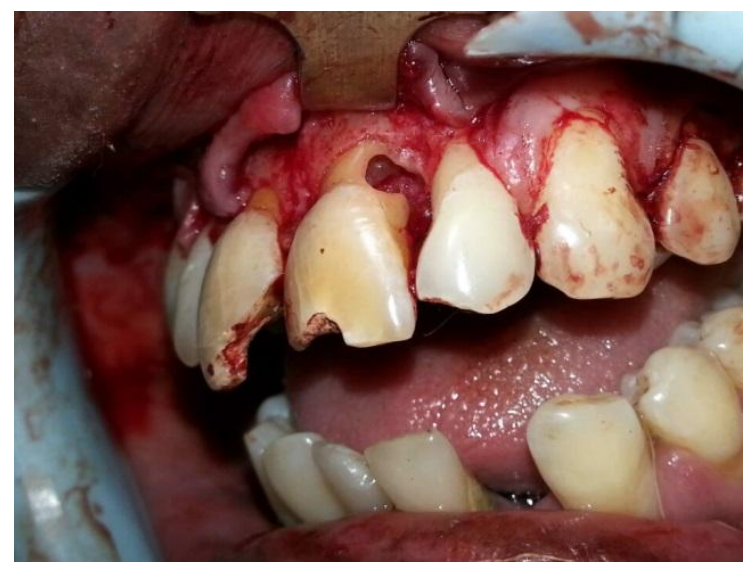

Figure 6: Complete removal of granulation tissue and exposure of resorptive defect

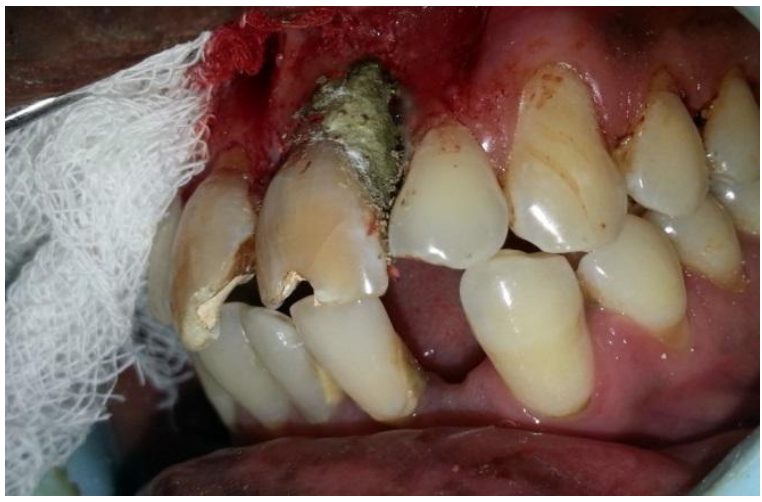

Figure 7: Resorptive defect sealed with MTA

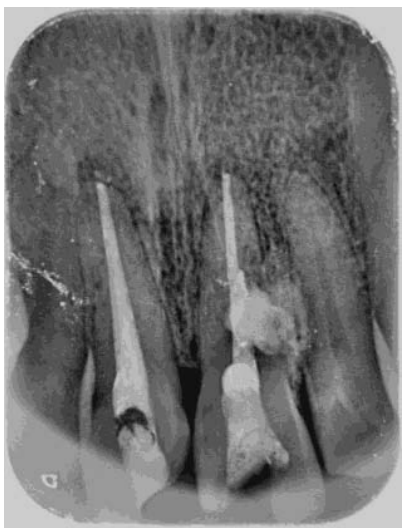

Figure 8: MTA placement intra operative view

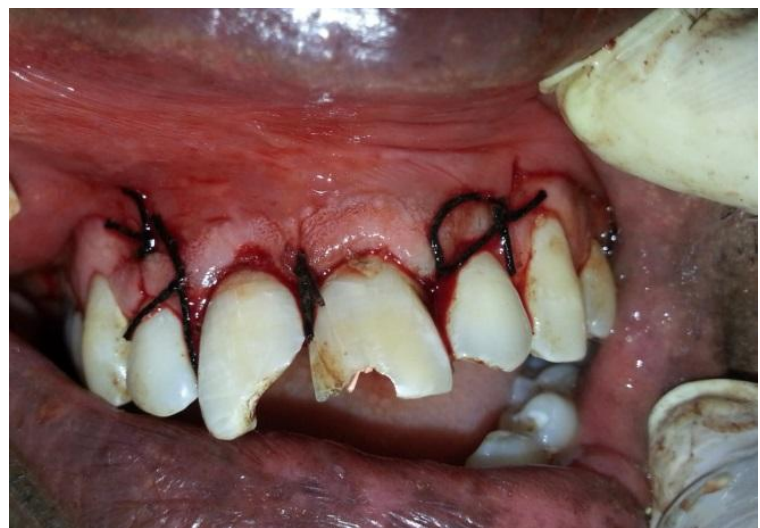

Figure 8: Sutures placed

Following this composite buildup of 11 and 21 was done. The patient was recalled after 1,3 , and 6 months for 


\section{International Journal of Science and Research (IJSR) \\ ISSN (Online): 2319-7064 \\ Index Copernicus Value (2013): 6.14 | Impact Factor (2014): 5.611}

clinical and radiographic follow-up. On clinical examination 21 was functional without sensitivity to percussion or palpation. The tooth showed normal physiologic mobility and no periodontal pockets on probing. The periapical radiograph showed regression in the size of periapical radiolucency with signs of osseous repair and no further progression of external resorption. There was initiation of osseous healing adjacent to the distal resorption site on the root surface.

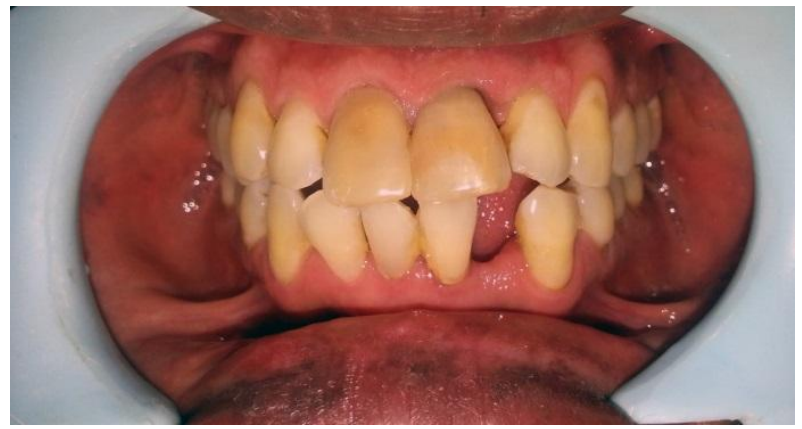

Figure 9: Six month follow up

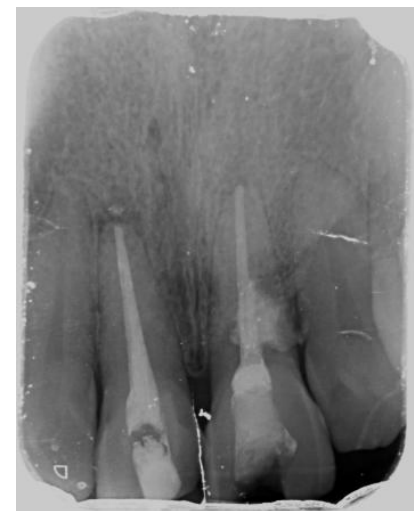

Figure 10: Six month follow up IOPA radiograph

\section{Discussion}

IERR is initiated by long term pulpal infections, resulting in the destruction of cementoblasts, loss of precementum and sometimes cementum in areas of the root surface. The resorptive process is then maintained by bacterial products from the infected root canal which provides the necessary continuous stimulation for the resorbing cells [5]. Thus, the treatment protocol for IERR should involve elimination of bacteria and their byproducts from the root canal system and dentinal tubules to stop the inflammatory processes involving the root surface to allow the regeneration of periodontium [12].

Cone beam computed tomography imaging (CBCT) can be very useful in treating root resorption. Periapical radiographs tend to underestimate the size of the resorptive lesion. However, CBCT scanning gives a more accurate estimate of the lesion size. The CBCT images also provide the precise location of the lesion in three dimensions and the relationship to the surrounding bone, which can be very helpful in decision making pertaining to surgical management of such lesions [13]. In this case, before considering surgery, $\mathrm{CBCT}$ imaging was used to locate the lesion accurately and to determine the relationship between the lesion and alveolar bone. The CBCT images showed that there was absence of labial cortical bone over the resorptive lesion, which was favorable for access to the resorptive defect and thus helped in the decision process of whether to attempt surgery.

Root lesions caused by external root resorption have been treated with different techniques and materials. In this case, after resorption lacuna was exposed surgically, the root canal treatment was subsequently performed because the root resorption was in relation with the root canal [14].

The conventional and preferred treatment protocol for a progressive IERR consists of chemomechanical preparation of the root canal system including a short-term dressing of creamy paste of calcium hydroxide to provide an alkaline $\mathrm{pH}$ within the root canal system and the dentinal tubules to kill the bacteria and neutralize the endotoxins, which are potent inflammatory stimulators [15], [16].

The antibacterial and tissue dissolution effect of sodium hypochlorite $(\mathrm{NaOCl})$ increases with its concentration, but this is accompanied by an increase in toxicity [17]. As the contact between the irrigant and the surrounding vital tissues cannot be completely avoided in cases of perforating resorption, judicious use of low concentrations of $\mathrm{NaOCl}$ is recommended. In this case saline rinse was done after each time $\mathrm{NaOCl}$ was used in order to neutralize its effects and avoid any caustic tissue reactions. Final rinse before obturation was with $2 \%$ chlorhexidene which has high substantivity and broad spectrum antibacterial activity which is more beneficial in retreatment cases like the present case.

Surgical management of IERR has generally involved periodontal flap reflection, chemo-mechanical debridement and restoration of the defect with amalgam, composite resin, or glass ionomer cement, and repositioning the flap to its original position [18]. Periodontal reattachment cannot be expected with amalgam or composite resin and is unlikely with glass ionomer cement, but there is experimental evidence to suggest that this might be possible if MTA is used in this situation [19].In addition, MTA inhibits the activity of bacteria [20], is not affected in the presence of moisture and blood, and also is able to harden and form a barrier because of its hydrophilic nature. Moisture in the surrounding tissue acts as an activator of a chemical reaction in this material [21]. In previous studies, MTA was successfully used as a barrier between the root canal space and the periodontal tissue in cases of root perforation in dogs [22] and humans [23]. Hakki et al [24] demonstrated that MTA does not have a negative effect on the viability and morphology of cementoblasts and induced biomineralization of cementoblasts.

In the case described here 1,3 , and 6 month radiographic recall showed osseous repair of periapicalpathosis. Although re-establishment of the entire periodontal apparatus did not occur, the resorption site remained stable throughout the 6 month follow-up period. The patient was asymptomatic, with the tooth exhibiting normal sulcular probing depth, mobility, and function. The patient was also 


\section{International Journal of Science and Research (IJSR) \\ ISSN (Online): 2319-7064}

Index Copernicus Value (2013): 6.14 | Impact Factor (2014): 5.611

pleased with the treatment outcome as a permanent tooth with otherwise hopeless prognosis was maintained.

\section{References}

[1] Leach HA, Ireland AJ, Whaites EJ, Radiographic diagnosis of root resorption in relation to orthodontics, British Dental Journal 2001; 190(1):16-22.

[2] Trope M, Blanco L, Chivian N, et al., The role of endodontics after dental traumatic injuries. In: Cohen S, Hargreaves KM, Pathways of the pulp. 9th ed. St. Louis, MO: Mosby-Elsevier; 2006:635.

[3] Opaci-Gali V, Zivkov S, Frequency of the external resorptions of tooth roots, SrpskiArhivzaCelokupnoLekarstvo 2004;132(56):152-6.

[4] St George G, Darbar U, Thomas G, Inflammatory external root resorption following surgical treatment for intra-bony defects: a report of two cases involving Emdogain and a review of the literature, Journal of Clinical Periodontology 2006;33(6):449-54.

[5] L. Tronstad, Clinical EndodonticsA Textbook, Theime Medical and Scientific Publishers, 2nd edition, 2002.

[6] A. Sigurdsson, M. Trope, N. Chivian, The role of endodontics after dental traumatic injuries, " in Pathways of the Pulp, S. Cohen and R. C. Burns, Eds., pp. 620-654, Mosby, St. Louis, Mo, USA, 10th edition, 2011.

[7] da Silveira HL, Silveira HE, Liedke GS, Lermen CA, Dos Santos RB, de Figueiredo JA, Diagnostic ability of computed tomography to evaluate external root resorption in vitro, Dentomaxillofacial Radiology 2007;36(7):393-6.

[8] ShivaniUtneja, GauravGarg, ShipraArora, SangeetaTalwar, Nonsurgical Endodontic Retreatment of Advanced Inflammatory External Root Resorption UsingMineral Trioxide Aggregate Obturation, Case Reports in Dentistry Volume 2012, Article ID 624792, 5 pages.

[9] Bergmans L, Van Cleynenbreugel J, Verbeken E, WeversM, Van Meerbeek B, Lambrechts P, Cervical external root resorption in vital teeth, Journal of Clinical Periodontology 2002;29(6):580-5.

[10] Carlos Estrela, Mike Reis Bueno, Ana Helena Goncalves De Alencar, RinaldoMattar, Jose' ValladaresNeto, Bruno Correa Azevedo, DDS, MSc, k and Cyntia Rodrigues De Arau'jo Estrela, Method to Evaluate Inflammatory Root Resorption by Using Cone Beam Computed Tomography, J Endod 2009;35:1491-1497.

[11]EmreAltundasar, BecenDemir, Management of a Perforating Internal Resorptive Defect with Mineral Trioxide Aggregate: A Case Report, J Endod 2009; 35:1441-1444.

[12]L. Levin and M. Trope, Root resorption, " in Dental Pulp, K. Hargreaves and H. Goodis, Eds., pp. 425448, Quintessence, Chicago, Ill, USA, 3rd edition, 2002.

[13]Richard S. Schwartz, J. William Robbins and Eric Rindler, Management of Invasive Cervical Resorption: Observations from Three Private
Practices and a Report of Three Cases, JOE Volume 36, Number 10, October 2010

[14] Ne R, Witherspoon D, Gutmann J, Tooth resorption. Quintessence International, 1999; 30 (1):9-25.

[15]Z. Fuss, I. Tsesis, and S. Lin, -Rot resorptiondiagnosis, classification and treatment choices based on stimulation factors, " Dental Traumatology, vol. 19, no. 4, pp. 175-182, 2003

[16] M. Trope, J. Moshonov, R. Nissan, P. Buxt, MC. Yesilsoy, "Short vs. long-term calcium hydroxide treatment of established inflammatory root resorption in replanted dog teeth, " Endodontics\& Dental Traumatology, vol. 11, no. 3, pp. 124-128, 1995.

[17] M. Zehnder, Root canal irrigants, "Journal of Endodontics, vol. 32, no. 5, pp. 389-398, 2006.

[18] HasanGuneyYilmaz, AtakanKalender, EsraCengiz, Use of Mineral Trioxide Aggregate in the Treatment of Invasive Cervical Resorption: A Case Report, JOE - Volume 36, Number 1, January 2010.

[19] Heithersay GS. Invasive cervical resorption. Endodontic Topics 2004;7: 73-92.

[20]Zhang H, Pappen FG, Haapasalo M, Dentin enhances the antibacterial effect of mineral trioxide aggregate and bioaggregate, J Endod 2009; 35:221-4.

[21] Lee SJ, Monsef M, Torabinejad M, Sealing ability of a mineral trioxide aggregate for repair of lateral root perforations, J Endod 1993;19:541-4.

[22] Holland R, Filho JA, de Souza V, Nery MJ, Bernabe' $\mathrm{PF}$, Junior ED, Mineral trioxide aggregate repair of lateral root perforations, J Endod 2001;27:281-4.

[23] Main C, Mirzayan N, Shabahang S, Torabinejad M, Repair of root perforations using mineral trioxide aggregate: a long-term study, J Endod 2004;30:80-3.

[24]Hakki SS, Bozkurt SB, Hakki EE, Belli S, Effects of mineral trioxide aggregate on cell survival, gene expression associated with mineralized tissues, and biomineralization of cementoblasts, $\mathrm{J}$ Endod 2009;35:513-9 